Bojan Cop

Ljubljana

\title{
ZUR MEDIOPASSIVEN 2. UND 3. PERSON DUALIS IM INDOGERMANISCHEN
}

Bekanntlich gibt es in einigen seltenen idg. Sprachen (im A ri schen, Griechischen, Tocharischen) auch Pexsonal. suffixe fïr die 2. und 3. P. Du. des Mediopassivs. Von diesen sind die griechischen Ausgänge - - -9ov(2. und 3. Du primär, 2. Du. sekundär), $-\sigma \vartheta r, y$, dor. $-\sigma \vartheta \ddot{\vartheta},(3$. Du. sekundär) sicher junge Neubildungen, s. z. B. Brugmann, Grdr. ${ }^{2}$ II 3 II, 657, \$ 602; Schwyzer, Gr. Gr. I 670, 672.

Ein voll ausgebildetes System solcher Endungen besitzt das Arische; im Tocharischen kommt nur eine einzige Form mit solcher Endung vor, die die 2.P. Du. Med. Imperativi darstellt. Unter solchen Umständen scheint es schwierig, aus diesem Material wahrscheinliche Schlüsse für das Urindogermanische zu machen. Und doch ist gerade die tocharische Form, die uns eine ausserordentlich grosse Hilfe leistet, wenn man sie richtig zu beurteilen vermag. Die tocharische Endung, verbunden mit den arischen, wird unter Einschluss der allgemeinen Tendenzen und Regelungen im System der indogermanischen Personalsuffixe ohne Schwierigkeit die urindogermanische Urform erscheinen lassen.

Das arische System dieser Endungen ist sehr bunt, dazu noch in verschiedenen Texten ziemlich verschieden.

Im klassischen Altindisch sind die Endungen, wie folgt:

a) 2. P. Du. Med.:

1) primär athematisch $-\bar{a} t h \bar{e}$;

thematisch (mit Themavokal vereint) $-\bar{e} t h \bar{e}$;

2. sekundär athematisch $-\bar{a} t h \bar{a} m$; thematisch (mit Themavokal vereint) -ēthām;

b) 3. P. Du. Med.:

1. primär athematisch $-\bar{a} t \bar{e}$;

thematisch (mit Themavokal vereint) $-\bar{e} t \bar{e}$;

2. sekundär athematisch -ätām;

thematisch (mit Themavokal vereint) -êtām. 
Diese ganz reguläre Konjugation ist schon im Vedischen die Regel, so dass ich die Beispiele aus den ved. Texten hole:

Ind. Präs. Med.: athematisch von ās $» \operatorname{sitzen\ll :~2.~Du.~} \bar{a} s-\bar{a} t h \bar{e}, 3$. Du.

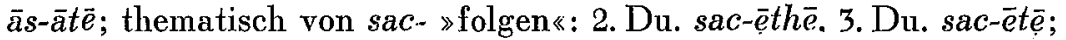

Ind. Perf. Med. von man-»denken« (athematisch): 2. Du. mamn-äthe

3. Du mamn-ätē ;

$s$-Aor. Ind. Med. und Inj. Med. (athematisch): von $s r j-~>e n t l a s s e n \ll$

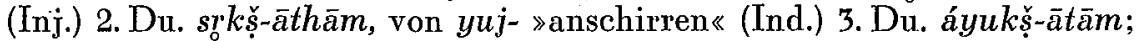

Imperf. Med. thematisch von $b \bar{a} d h-» d r a ̈ n g e n \ll ~ u n d ~ r i j-(r \bar{e} j-)$ »beben《

2. Du. ábādh-ēthām, 3. Du. árēj-ètām.

Genau dieselben Formen sind fürs klassische Altindisch anzusetzen. Zu den klass. Endungen s. z. B. Thumb-Hauschild, Hb.d. Sanskrit II ${ }^{3}$ 208 f., $\$ 432$; zum Optativ ebda. 214 f., $\$ 437$ Ende.

Die eben dargelegten Endungen sind ohne Zweifel Produkt langer Entwicklung mit der Tendenz zu einer ausgewogenen Systematisierung. In einigen recht seltenen Fällen schimmert die ältere Bildung dieser Formen auch im Vedischen noch durch: ${ }^{1}$

Aor. 3. Du. Med. ádh-ītām »sie beide setzten«, das aber auch in ádhī-tām mit der Endung -tām (dann vielleicht aktiv?) und den Stamm. -dhi- wie in 1. Pl. Med. adhïmahi, dhïmahi (=Ind. und Inj.) zerlegt werden kann! ${ }^{2}$

nach Macdonell, Ved.Gr. (1910) 343, § 460 ist Imper. Präs. 2. Du. Med. die Form dìdhi-thàm. (AV.), nach anderen = Opt., von $d \bar{i}-d h \bar{\imath}-$ $\gg$ Acht haben $;^{3}$

Opt. des s-Aor. 2. Du. Med. ist trä-s-i-thām »möget ihr beide beschützen «; vgl. Macdonell 381, § 525;"

$c i k \bar{e}$-the $\bar{e}$ ist die 2. Du. Ind. Perf. Med. von $c i-»$ bemerken « (Macdonell 359, für reguläres *ciky-ä'thē); $;^{5}$

hierher auch $\operatorname{tr} \bar{a} ' s-\bar{a}-t h \bar{e}$ als 2. Du. Konj. s-Aor. Med. (vgl. oben trā'sīthām zur Bed.); Macdonell 380, § 523 (für *trā'sāithèe, s. noch unten) $;^{6}$

ebenso ist wohl aufzufassen $r d h-\bar{a}-t h \bar{e}=2$. Du. Konj Aor. Med. (ogl. von derselben Wurzel 3. Sg. Akt. Konj. Aor. fodhat!) von rodh-»gedeihen«, von Macdonell 369, §504 fälschlich als Opt. Med. definiert; ${ }^{6}$

1 Eine Ưbersicht der unregelmässigen altindischen Formen der 2. und 3. Du. gibt Bartholomae, KZ. 29, 1888, $283 \mathrm{ff}$.

2 Bartholomae, KZ. 29, 284 f. schwankt zwischen der Analyse a-dh-ìtām und a-dhi-tām, zieht jedoch die erstere Möglichkeit vor: Suffix -itām.

3 Bartholomae a. O. 284 gibt dieselbe Analyse, Suffix -thām.

4 Bartholomae 284 schwankt zwischen tra's's-thām (Opt.) und trā's-ìthām (Inj.), doch scheint ihm der Inj. die bessere Lösung, Suffix demnach -īthām.

5 Vgl. Bartholomae 284.

- Auch Bartholomae 287 nimmt $r d h a \bar{a}-t h \bar{e}$ als Konj. wie trāsāthē; Suffix nach ihm entweder -āth $\bar{e}$ oder -ăthè 
wundererregend ist auch 2. Du. Imper. $s$-Aor. Med. rāa-s-āthām von rā- »verleihen, gewähren «; die übrigen zwei Formen dieses Imperativs, 3. Sg. Med. rās-a-tām und 3. Pl. Med. rās-a-ntām, zeigen, dass es sich um th e m a tis che Flexion handelt (vgl. Macdonell 381, §526); demnach war ein $-\bar{a}-t h \bar{a} m$ urspr. auch in der thematischen Flexion möglich? oder gegen die andern zwei Formen trotzdem athematisch? (oder sogar Inj., vgl. $s r k s \grave{s}-\bar{a} t h \bar{a} m$ oben?).

Die eben vorgeführten Formen zeigen klar, dass die beiden Vokale $-\bar{a}$ - und $-\bar{e}$ - (aus Themavokal $-\breve{a}-+{ }_{-}-$-) urspringlich nicht obligatorisch waren, m.a.W., es gab auch kürzere Personalendungen, die mit Dental anlauteten:

2. Du. primär -the $\bar{e}$, sekundär -thām;

3. Du. primär"-tê, sekundär *-tām (beide unbelegt, was für weitere Forschung, s. unten, wichtig sein kann).

Man hatte also urspsünglich drei Reihen von Endungen für die untersuchten zwei Personen:

2. P. Du. primär -the,$-\bar{a}-t h \bar{e}$ und $*_{-\bar{t}-t h e}$;

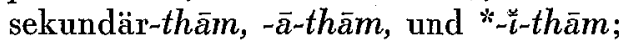

3. P. Du. primär ("-têe?), $-\bar{a}-t \bar{e}$ und * *

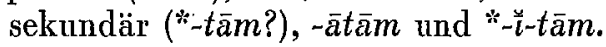

Man kann nur über die Quantität des $-\breve{l}$ - in der letzten Reihe zweifeln, denn es kommt (wenn man ádh-ïtām nicht anerkennt) nur in Verbindung mit dem Themavokal vor, s. oben; dasselbe gilt auch von $\mathrm{K}$ o $\mathrm{n}$ ju $\mathrm{nktiv}$, wo jedoch auch in der athematischen Konjugation normal langer Moduszeichen gebraucht wird; so haben wir hier -āithe für die 2. Du. Med., -āitē für die 3. Du. Med.:

a) thematische Konjugation: 3. Du. yát-āitē (yat-»streben«), 2. Du. $p r n-\bar{a}^{\prime} i t h \bar{e}$ (prn-»füllen«) u.a.;

b) athematische Konjugation: 2. Du. dhāithē (dhā-, Aor. á-dhā-t, »setzen u.sw.«), 3. Du. bráb-āitēe (brū- »sagen«) usw.

Die einzige Ausnahme aus der athematischen Konjugation, dhe'the ${ }^{7}$ 2. Du. Konj. Aor. Med. von $d h \bar{a}-$ (daneben dhāithēe, ist unklar, denn dhāithe ist hier eigentlich das einzig Mögliche: $d h \bar{a}-+$ Konj.-Zeichen $-\breve{a}-+{ }_{-}^{*}$ thai kann nichts anderes ergeben (Macdonell $369^{3}$ spricht von einer $\gg$ transfer form for * $d h \bar{a}$ the $\ll$; ?).

Der Langrokal als Konjunktivzeichen auch in der athematischen Konjugation ist viell. mit $-\bar{a} i$ der 1. P. Sg. Med. Konj. vergleichbar;

7 Bartholomae 287 nimmt merkwürdigerweise kein Wunder an dieser Form, die nach ihm zum Typus auf -èthe gehört, den er auch im Konj. parēthe (var»wählen «) wiederfindet. Im letzteren ist regelrecht kurzes Konjuktivzeichen gebraucht, das sonst von $-\bar{a}-$ auch in der althematischer Konjugation verdrängt, wurde. Vgl. Anm. 9. 
etwas daraus für die Urform der Personalendung (etwa auch *-ăi-thăi?) zu postulieren wäre zu gewagt.

Die oben festgestellte dreifache Buntheit der fraglichen Personalendungen im Vorvedischen wird durch die Tatsachen des Avestis che n aufs schönste bestätigt. Hier finden wir (hauptsächlich nach Bartholomae, Grdr. d. iran. Phil. I 1, 66, § 120). ${ }^{8}$

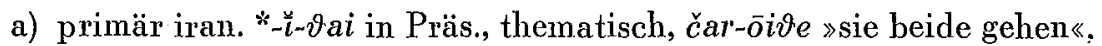
zu ai. 2. Du. $-\bar{e} t h \bar{e} ; 9$

b) primär iran. *-tai, und zwar:

1. thematisch gäth. myăsai-tē »sie beide mischen sich «, Präs.;10

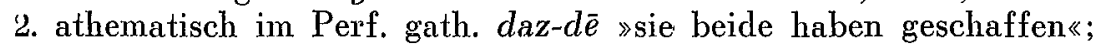
aus *dhadh-tai; 11

c) primär iran. *-ă-tai athematisch im Präs. gāth. parono-aitēe »sie beide wählen «; ${ }^{12}$

d) primär iran. *-ä-tai athematisch im Perf. gāth. maman-äitē »sie

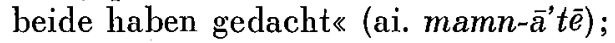

e) primär iran. *-ut-tai, thematisch;

1. im Präs. Ind. vīs-aète »die beiden kommen«; ai. 3. Du. -ète ;

2. im Konj. der athematischen Konjugation gāth.jam-aête $»$ sie beide sollen kommen« gegen ai. bráo-äitēe (s. oben), das sekundär ist nach Bartholomae a.O. 66 Anm. 3;

f) sekundär iran. *-a-turm athematisch gath. asro-ātam »sie beide wurden gehört« (ai. dagegen -ătām);

g) sekundär iran. *-ü-tăm, und zwar;

1. athematisch $d a^{i} \sim \delta$-it

2. thematisch im Imperf. aparas-aètam »sie beide beredeten sich«, gegen ai. -ètām.

$8 \mathrm{Vgl}$. schon Bartholomae, KZ. 29, $285 \mathrm{f}$.

9 Vgl. auch Bartholomae, KZ. 29, 287. Er zieht heran noch den av. Konj. isöive von is- „Herr sein über (gehört als zum athemat. Präs. gehörend zum. Konj.-Typus von Anm. 7). Doch hält Bartholomae, Altiran. Wb. 26 isōive augenscheinlich fuir Form des thematischen Ind. Präs. isa-.

10 Vgl. Bartholomae 286, der aber als Suffix hier -aite ansetzt, offenbar weil er an athemat. myās-denkt. Als myāsa- wird jedoch von ihm der Präsensstamm später angesetzt, vgl. Altiran. Wb. 1190.

11 Vgl. Bartholomae 285. Das Suffix *-tai findet Bartholomae 285 (und 287) auch im Altind.: 3. Du. Präs. Med. von patya- soll patya-tē RV. III 54,8 sein: »sie beide haben in ihrer Gewalt«; ferner 3. Du. Konj. Aor. Med. von yam- soll yama-të RV. VII 37,3 sein: »die beiden werden schenken «. Spätere Abhandlungen lassen diese zwei Formen ausser Acht. Bei Macdonell 369 findet sich yamate iiberhaupt nicht, wohl aber zitiert er sie als 3. Sg. Ind. Präs. Med. auf S. 321.

$12 \mathrm{Vgl}$. Bartholomae $285 \mathrm{f}$. Nach Geldner sollten hierher noch die drei av. Formen gehören: daখaite, marazaite, čaraite, sind aber nach Bartholomae 286 zu unsicher. - Aus dem Altindischen führt Bartholomae $286 \mathrm{f}$. Formen aus Rgveda, die zwar mit dem Ausgang-ăth-, -āt- iberliefert, von den Dichtern aber nach Ausweis des Metrums mit kurzer Pänultima gesprochen worden sind:

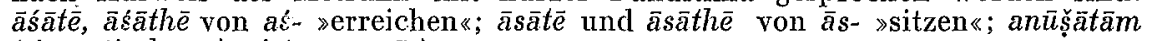
(sigmatischer Aorist von nī-). 
Alle avestischen Formen dienen als die 3. Du., auch die unter a) angefuihrte, die ja urspriinglich 2. Du. war $\left(-\vartheta_{-}=\right.$ai. $\left.-t h-!\right)$; man weiss übrigens, dass auch im Akt. alle Formen, auch diejenigen mit ar. -th- =

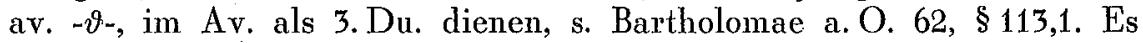
handelt sich um eine erst iranische Neuerung, die aus dem Akt. stammt, wo in gewissen Stellungen urar. $-t$ - und -th- zusammenfielen und so einen Promiskue-Gebrauch nach sich zogen.

Im Av. haben wir noch einen vierten Typus der Dualendungen: mit kurzem Vokal a- vor dem Dental, s. Pkt. c)! Vielleicht ist dieser Typus im Altind. in den Konj.-Formen tra's sāthe und rodhāthe (vgl. Bartholomae, KZ. 29, 287), ferner im Imper. rāsāthām erhalten, wobei das dem Dental vorausgehende $-\bar{a}-$ in den Themavokal (bzw. Konjunktivzeichen) + unser kurzes -a- (Suffixbestandteil) zu zerlegen ist. ${ }^{12}$-- Übersicht der urar Typen:

\begin{tabular}{|c|c|c|c|c|c|}
\hline & & Typus I & II & III & IV \\
\hline 2. Du. & $\begin{array}{l}\text { primär } \\
\text { sekundär }\end{array}$ & $\begin{array}{l}\text { *-thai } \\
*-t h a \bar{m}\end{array}$ & $\begin{array}{l}\left({ }^{*}-a ̆ t h a i\right) \\
(*-a ̆ t h a m)\end{array}$ & $\begin{array}{l}\text { *-āthai } \\
\text { *-āthām }^{2} \text { - }\end{array}$ & $\begin{array}{l}\text { *-itthai } \\
\text { *-i }^{2} \text { thām }\end{array}$ \\
\hline 3. Du. & $\begin{array}{l}\text { primär } \\
\text { sekundär }\end{array}$ & $\begin{array}{l}{ }^{*}-t a i \\
("-t a ̈ m)\end{array}$ & $\begin{array}{l}\text { *ătai } \\
(*-a ̆ t \bar{a} m)\end{array}$ & $\begin{array}{l}*-\bar{a} t a i \\
*-\bar{a} t \bar{a} m\end{array}$ & $\begin{array}{l}{ }^{*}-\bar{t} t a i \\
{ }^{*}-\bar{t} t a \bar{a} m^{13}\end{array}$ \\
\hline
\end{tabular}

Die in Klammern gesetzten Endungen sind nicht belegt oder nur unsicher zu exschliessen. Es ist fraglich, ob sie jemals gebildet wurden, obwohl das Gleichgewicht des Systems sie erheischt.

Im Avestischen ist ausser der Promiskuität der beiden Dualpersonen auch noch eine weitere Neuerung zu beobachten: in den Sekundärendungen kommt $-\breve{a} m$ statt des langen -àm vor, wohl nach der Aktivendung-tom (aus *-tăm), die im Av. als 3. Du. dient; vgl. Bartholomae a. O. 66, § 120 Anm. 4.

So sind die arischen Endungen für die 2.-3. P. Du. zwar sehr bunt, jedoch in den meisten Teilen leicht erklärbar:

a) die Opposition -th- für die 2. Du.: -t- für die 3. Du., die im Altindischen ganz klar und regelmässig hervortritt, hat auch sonst klare Anhaltspunkte, so in den primären Endungen dex 2.-3. Du. Akt.: ai. 2. Du. bháva-thas (Ind. Präs. Akt.), 3. Du. bháva-tas; in den Perfektendungen der 2.-3. Du. Akt.: 2. Du. Ind. Perf. Akt. ai cakr-áthur »ihr beide habt gemacht«, 3. Du. cakr-átur; in der 2. P. kommt -th-auch sonst vor: in der 2. Sg. Ind. Perf. Akt., idg. "tha: ai. $v \bar{e}^{\prime} t-t h a=$ gr. oĩ $\sigma-\vartheta x_{-}$du

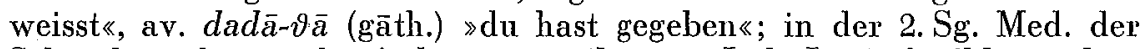
Sekundärendung: idg. " - thêes $=$ ai. -thās im Ind. Imperf. ábhava-thās,

13 Mit dieser Tabelle stimmt die Übersicht bei Bartholomae $286 \mathrm{f}$. vollkommen überein, nur führt er als besondere Formen noch die av. mit kurzem -avor $-m$ an. Die hat ex später als selkundär erklärt, sieh im Text. Er führt hier

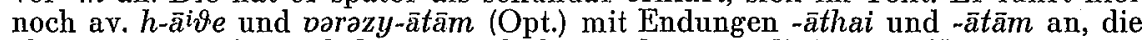
aber sonst im Av. unbekannt sind. Später lässt er diese zwei Formen ausser Acht. varəzyātām liest er später nach K 7 a als -atām mit kurzem erstem -a(Altiran. Wb. 1376 mit Anm. 9). 
Opt. bhávē-thās; schliesslich in der 2. P. Akt. der Primärendung, wenigs-

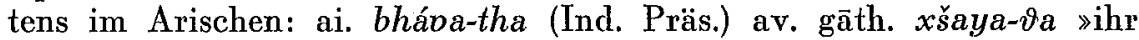
herrscht «; die 3. P. hat bekanntlich -t- auch sonst, so in der 3. Sg. in den idg. Endungen (Alkt.) *-t, *-ti, (Med.) *-to, *-toi; im Du. in der 3. Du. Akt. *-tām. Leider kommt -t- auch in der 2. P. im Pl. vor: idg. *-te in ai. Imperf. Akt. ábhana-ta usw.

b) die Ausgänge *-thai und *-tai mit Varianten zeigen dieselbe Diphthongierung wie die mediopassiven Endungen 1. Sg. *-a ${ }^{x} i$ (ai. bháo-êe, 2. Sg. *-soi (ai. bhába-sē, gr. ark. xeî-ol »du liegst«), 3.Sg. "-toi (ai.

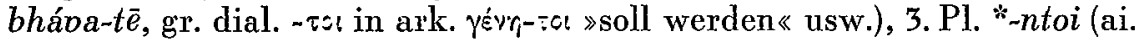

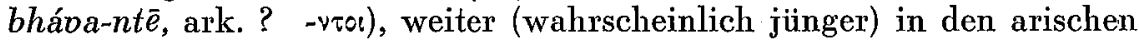
Endungen 1. Du. ai. - - ahe , 1. Pl. ai. $-m a h \bar{e}$, av, $-m a^{i} d e$ und 2. Pl. ai. $-d h v \bar{e}=$ av. gatth. $-d u y \bar{e}$, jung. $\sim \vartheta r e$; alle diese Endungen, auch die uns beschäftigenden dualischen, sind primär; vgl. auch perfektische Ausgänge 1. Sg. *-ai, 3. Sg. *-ei, 3. Pl. *-roi.

c) das *- $\bar{a}^{x} m$ der sekundären Endungen *thām, *-tām kann unmittelbar mit *-ām der aktiven Endung 3. Du. gleichgesetzt werden (idg. *-tām), doch ist das nicht obligatorisch, denn unsere Endungen können auch *-óm oder "-e $m$ enthalten. S. noch unten.

Es sind also eigentlich nur die vokalischen Elemente vor den Dentalen, ar. *-ă-, *-ā und ${ }^{*} \breve{l}-$, die unerklärt geblieben sind. ${ }^{14}$ Wenn wir das Mittel, sie zu erklären, finden, dann ist unsere Aufgabe ehrlich erfüllt.

14 Schmidt, KZ. 26, 12 findet in -ithe $-i$ - als Schwächung von $-\bar{a}-$; das hat schon Schulze, KZ. 27, 427 widerlegt; $-i$ - wäre im Idg. als -a- anzusetzen und hinter Vokal unmöglich. Schulze selbst setzt als starke Stufe -āithē (daraus später $-\bar{a} t h \bar{e})$ an, als schwache $-\bar{i} t h \bar{e}:$ vgl. váhethè: dvišathé. Nach Bartholomae, $K Z .29,284$ ist aber auch Schulzes Erklärung unzureichend, da wir auch die dritte Reihe dieser Dualendungen beruicksichtigen müssen, die nur mit Dental beginnen, wie -the , -té, oder mit kurzem Vokal anlauten (-athes, -até). Mit -äithe usw. können die letzteren nicht erklärt werden.

S. 288 nimmt Bartholomae an, dass die verschiedenen ursprünglichen Suffixe sich gegenseitig beeinflusst haben. Er fährt fort: »So viel aber lässt sich mit hoher Wahrscheinlichkeit behaupten, dass jedes der vier Suffixe -2 . Person, 3. Person, primär, sekundär - ursprünglich zwei verschiedene Formen, aber auch nicht mehr als zwei, gehabt hat, eine betonte und eine unbetonte. Betont, so nehme ich an, hatten die einen den Anlaut $\bar{a} i$ (daraus dann später $\bar{a}$ ), die andern $a$; unbetont die ersteren $\bar{l}$, die letzteren $O$; von den Sekundärsuffixen hatte das eine kurzen, das andere langen Vokal vor dem schliessenden $m$. Standen z. B. in der Ursprache nebeneinander die Formen ( - die Qualität der aVokale ist ohne Bedeutung -): *strnrō'tai, *strnnitai, *bhéreitai und anderseits: *strntoáthai, *strnuthai, "bherethai: so wäre das ai. strnoäthe eine Neubildung

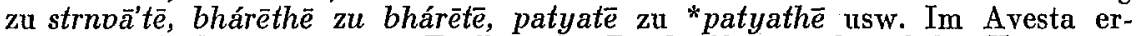
strechte sich der gegenseitige Einfluss der Dualsuffixe auch auf den Konsonantismus; in den jüngeren Teilen desselben steht z. B. čarōive im Sinn des ai. cárētē.....

Wir werden sehen, dass ein Ablaut in der Pänultima unserer Suffixe kaum anzunehmen ist; auch erklärte er nur einen Teil der Endungen, $-\bar{a}-$ und $-a-\operatorname{der}$ ersten Silbe derselben blieben noch immer ohne Erklärung.

Doch blieb der Ablaut als ein beliebtes Mittel zur Erklärung unserer Endungen in den späteren Jahren in Mode. 
Nun scheint die Antwort auf diese Frage im Tocharis chen zu liegen. Denn diese Sprache, genauer der Dialekt B, kennt für die 2. Du. Imper. Med. eine Endung - ai-t, die laut Krause-Thomas, Toch. El. I 177, 256 und $260^{15}$ einzig in B p-yam-tts-ait »macht ihr beide! « (von yām$\gg$ machen $\ll$ ) vorliegt und noch unerklärt ist; über die scheinbar ähnliche aktive 3. Du. Prät. B ai-s vgl. kurz unten.

Das auslautende - $t$ - dieser Endung ist doch wohl dem - $t$ der Endung für 2. Pl. Med. Prät. und Imper. in B identisch, vgl. Imper. p-yām-ts-a-t »macht! . Die beste Erklärung für diese Endung ist die von Pedersen ${ }^{16}$ : aus idg. *-dhu zu ai. $-d h v a,-d h v a m$, gr, $-\sigma \vartheta \varepsilon$; es ist also eine pluralische Endung, die in den Dual eindrang. Was jedoch vor ihr steht, d, h. der Diphthong -ai-, kann nicht pluralisch sein, denn dort finden wir im Imper. -a-, s. oben.

Dieser Diphthong muss irgendwie in Zusammenhang mit dem arischen Diphthong einiger Endungen, die oben erörtert wurden, stehen: mit ai. $-\bar{e}-$ in $-\bar{e}-t h \bar{e},-\bar{e}-t \bar{e},-\bar{e}-t h \bar{a} m,-\bar{e}-t a \bar{m}$, mit av, $-\bar{o} i-$ in $-\bar{o} i-\vartheta \vartheta,-a \bar{e}-$ in $-a \vec{e}-t e,-a \bar{e}-t a m$, mit ai. $-\bar{a} i-(\mathrm{Konj}$.) in $-\bar{a} i-t \bar{e}$. Es ist zwar fraglich, ob auch der Vokal -a- des toch. Diphthonges mit dem ersten Bestandteil des arischen Diphthonges zu identifizieren ist, denn als Nebenzeichen des Imperativs kommt im Tocharischen in sonstigen Formen der Vokal sa- vor: B Akt. 2. Sg. (p-)kāl-a, 2. Pl. (p-)kal-a-s(o), Med, 2. Sg. (p-)kal-a-r, 2. Pl. (p-)kal-a-t von kal- »führen, bringen « (in A im Med. 2. Sg. pa-kl-ā-r, 2. Pl. po-kl-a-čc). u.a. Es ist also am besten, wenn wir das -ai- von B $-a i-t$ in dieses imperativische $-a-+$ ein $i$-Element zerlegen. Erst das so gewonnene $i$-Element muss mit dem im Arischen in Hülle und Fülle vorkommenden $i$-Element der dualischen Endungen identifiziert werden: ausser in den oben eben vorgestellten $i$-Diphthongen noch selbständig in den Endungen ai. -ì-tām (in ádh-ītām?) und av. -ìtam.

Das Arische und das Tocharische stimmen also wenigstens in dem eben hervorgeholten $i$-Element überein: eine Schicht der mediopassiven Dualendungen in der 2.P. und 3. P. hatte also im Indogermanischen offenbar als éines der Kennzeichen ein -i- (nach dem Arischen zu urteilen doch wohl lang) im Gebrauch.

Wackernagel, Altind. Gr. I 36: das -i- in -ēthe, $-\bar{e} t \bar{e}$ lautet mit -ā- von -āthe, $-\bar{a} t \bar{e}$ ab; dasselbe S. 89 , wo er konstatiert, dass das $-\bar{l}-$ in der thematischen Konjugation im Nachton steht, während $-\bar{a}^{\prime}-$ betont ist.

Reichelt, Aro. El. 131 nimmt, ganz wie Bartholomae, Ablaut nicht nur für das Verhältnis - $\bar{a}-:-\bar{\imath} \sim$, sondern auch für das Paar ${ }^{*}$-atai: *-tai an. Ebenfalls denkt an Ablaut Thumb-Hauschild, Hb. $d . S k r .^{3}$ II 209.

Die gleiche Erklärung bei Brugmann, Grdr.2 II 3 II, 657, nur ist er vorsichtiger: für uridg. *- ${ }^{*}-$ an Stelle des ar. $-\bar{a}-$ vor dem Dental ist sonst kein Anhalt.

15 Vgl. noch Krause Westtoch. Gr. I 26, 197, 202, 272. Auch hier S. 202 denkt Krause in betreff des Diphthonges -ai- an die aktive 3 . Du. Prät, -ai-s; vgl. unten im Text.

${ }_{16}$ Pedersen, Z. toch. Sprachgesch. 6 f.: toch. $-t$ aus ${ }^{*}-d h u$. Doch ist phonetisch auch *-dhum möglich. Vgl. Čop, Lingu. V, 1963, 45 Anm. 56. 
Betrachten wir nun die iibrigen vor dem Dental der fraglichen Dualendungen stehenden Vokale, so bekommt man den Eindruck, dass vor uns einfach di e nom inalen Dualendungen liegen:

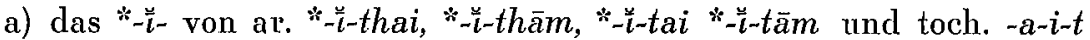
erinnert ja augenscheinlich an das dualische Nominalsuffix $*_{-\breve{l}}$ und $*_{-\bar{\imath}}$

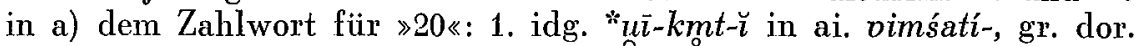

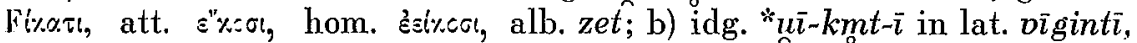
akymr. uceint; c) unklar av. vissaiti, arm. khsan; urspr. Dual »zwei-Dekaden $i^{17}$ b) im Nom.-Akl. Du. der Feminina auf $-\bar{a}-:^{18}$ idg. . *ai (mit Stosston) aus $*_{\sim a} H-\bar{l}$, z. B. in ai. áśpe $»$ Stuten«, av. urva ${ }^{i} r e » P f l a n z e n \ll$, ir. tūaith »Völker《 aus "tōtī < toutai, lit. rankì = sl. rọcě »Hände《; c) im Nom.-Akk. Du. der Neutra aller Stämme:19 o-Stämme idg. " $-o-i$ (Stosston!) aus " $-o-\bar{\imath}$ in ai. $y u g \bar{e} \vec{e}=$ sl. izě » Joche , konsonantische Stämme in ai. $d h \bar{a}^{\prime} m(a) n-\bar{\imath} \gg$ Satzungen $\ll, ~ s l . ~ i m e n-i \gg N a m e n \ll$ usw. usw. Auch hier ist * ${ }^{*}$, wie wir unter a) sahen, kurz oder lang, wie vielleicht auch in der verbalen Dualendung.

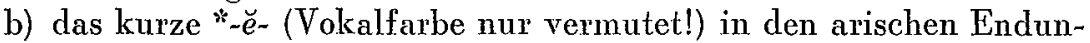

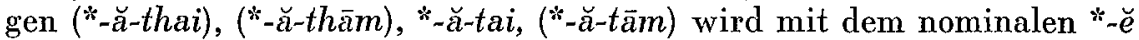
des Nom.-Akk. Du. Mask. und Fem. der konsonantischen Stämme iden-

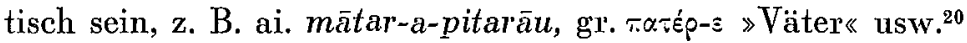

c) das lange " $-\bar{e}-$ (Vokalfarbe nur vermutet!) in den arischen Endun-

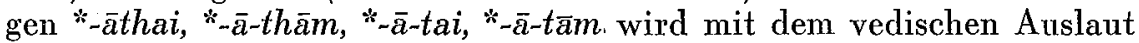
des Nom.-Akk. Du. Mask. und Fem. der konsonantischen Stämme -ā identisch sein, vgl. ai. ved. pitár-âa $\gg$ Väter《 usw. ${ }^{21}$

Es ist klar, dass unter diesen Umständen keine Möglichkeit der Iden-

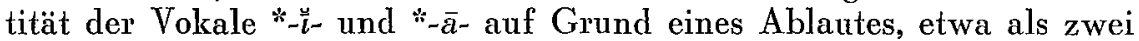
Ablautstufen eines urspr. Langdiphthonges ${ }^{*} \bar{a}^{x} i$, besteht, gegen die häufige Erklärungsweise dieser Vokale, die z. B. noch bei Thumb-Hauschild, $H b$. d. Sanskr. II ${ }^{3} 209$ zu finden ist. Gegen diese Erklärung spricht ja schon das kurze -ă- in ar. *ă-tai, ferner der Umstand, dass die Endungen mit *-ّu- fast ganz auf die thematische Konjugation beschränkt sind (es sollte gerade in der athematischen Konjugation die Alternation *-āi-: *-ā-: "-i- erwartet werden). ${ }^{22}$

Wenn aber die Identität dieser Vokale mit den nominalen Endungen des Nom.-Akk. Du. angenommen wird, so muss man eigentlich nachweisen, dass wenigstens ein Teil der Formen des Verbum finitum nominalen Ursprunges ist. ${ }^{23}$ Das ist heute

17 S. zu den Formen Pokorny, Idg. EW. 1177.

18 S. Brugmann, Grdr.2 II 2, $198 \mathrm{f}$. mit Weiterem.

19 S. Brugmann, Grdr.2 II 2, $201 \mathrm{ff}$.

20 S. Brugmann, Grdr. ${ }^{2}$ II 2, 200 f.; weiter z. B. Specht, Urspr. d. idg. Dekl. 311 mit Lit.

21 Brugmann a. O. $200 \mathrm{f}$.

$22 \mathrm{Vgl}$. Anm. 14 oben.

${ }^{23}$ Aber sogar dieser Nachweis ist nicht obligatorisch. Denn es ist sehr wahrscheinlich, dass die idg. Numerusbildungen einst ebenso gleichmässig beim 
wohl kein Geheimnis mehr; denn nicht nur im Verbum finitum des Semitischen und des Uralischen z. B., sondern auch im Verbum finitum des Indogermanischen kommen klare nominale Gebilde vor, so vor allem die 3. P. Pl. mit der Endung -nt usw., die ja mit dem Partizip auf $-n t$ identisch sein muss, obwohl es nicht klar ist, wie sie zur pluralischen Geltung gekommen ist (es fehlt in ihr jedes Pluralzeichen!). Auch die 3. Sg. mit der Endung * $t$ usw. ist nominal: man vergleicht ja öfters und mit vollem Rechte die Nomina agentis auf $-t-$, z. B. ai. -stú-t- »lobpreisend $\ll$.

Unsere Dualendungen stellen also in ihrem Vokalanlaut eigentlich alte Nominalendungen vor. Man fragt sich dann, wie es dazu kam, dass sich an die sicherlich urspr. schon fertigen Formen auf $*_{-\breve{l}, ~},-\breve{e}$, $" \vec{e}$ noch die einen Dental enthaltenden Formantien anschlossen, die dann die so komplizierten Gebilde zustande brachten. Hier muss man etwas weiter ausholen.

Das Urindogermanische besass einst $\mathrm{zwei} g \mathrm{rundverschie-}$ dene Reihen von Personalendungen, die man an der Hand hethitischen Teilung der gesamten Verbalflexion in die mi-Konjugation und $h i$-Konjugation am besten mi-Reihe und $h i$-Reihe nennt. Im Sg. (1., 2., 3. P.) und in 3. P. Pl. waren diese zwei Reihen durch folgende Formantien vertreten:

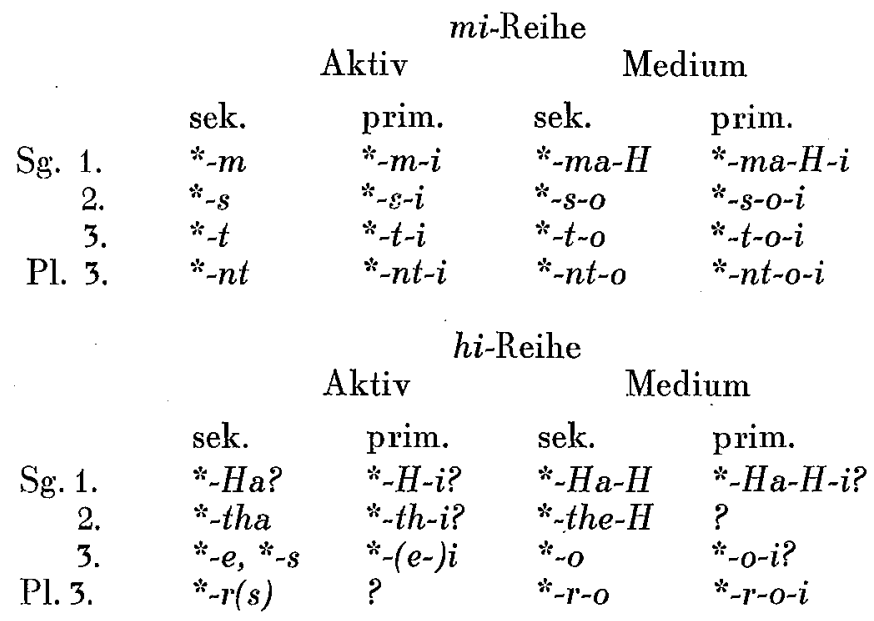

Nomen wie beim Verbum mit gleichen Engungen geformt waren, wie dies im Uralischen der Fall ist.

Es ist also anzunehmen, dass grundsätzlich auch das idg. Verbum dieselben Endungen wie das Nomen verwendete. Erst nachträglich kam es zu Verschiedenheiten im Bau der Endungen, da das Verbum durch innere Ummodelungen und gegenseitige Einflüsse sowie neue Bedürfnise (z. B. Tempus) eigene Wege einschlug. 
Jede Auseinandersetzung mit den bisherigen Ansichten in einzelnen Fällen sowie jede Erklärung der oben vorgelegten Ansätze würde den Rahmen dieses Aufsatzes sprengen. Ich spare weitere Auslegungen für spätere Gelegenheiten, bemerke jedoch hier, dass die hi-Reihe im Sg. sicherlich zwei verschiedene Gruppen von Endungen besass, von welchen oben nur die wichtigsten genannt wurden.

Der Unterschied zwischen der mi-Reihe und der hi-Reihe ist zwar am klarsten in der 1.P. Sg., doch scheint am wichtigsten die 3. P. Sg. zu sein: hier ist für die mi-Reihe die Anwesenheit des t-Lautes im Anlaut der Personalendungen bezeichnend, fiir die hi-Reihe dagegen die Abwesenhei desselben La tes. Nun ist es bekannt, dass die Personalendungen der 3. Du. im »Brugmannschen « Indogermanisch mit $-t$ - anlauten; so wenigstens im Aktiv. das ja einzig klar ist: primär "*t-es, sekundär *-tām (vgl. Brugmann, Grdr." II 3 II, 640). Es ist nun klar, dass wenn wir unsere Erkenntnisse aus der 3. Sg. auf die 3. Du. anwenden, auch hier ursprünglich zwei Reihen von Endungen, je nach der Konjugation, bestanden; denn es ist augenscheinlich, dass das $-t$ - von *-t-es und *-t-ām identisch mit dem -t- der mi-Endungen der 3. Sg. ist; somit sind "-t-es und *-t-ām ursprünglich Endungen der mi-Konjugation. Neben diesen muss es demnach einst auch Endungen der hi-Konjugation gegeben haben, die nach dem Verhältnis im Sg. ohne - $t$ - anlauteten. Diese rein theoretische Folgerung wird wenigstens zum Teil durch die tocharische aktive Endung der 3. Du., B ais, bestätigt. Sie wird zwar eingehender an einer anderen Stelle erklärt werden, hier nur das Wesentliche: das -s kommt auch sonst in tocharischen Personalendungen vor, ist also eine tocharische Zutat; das ubriggebliebene -ai- lautet mit Vokal an, ist schon auf Grund dieses Merkmales der hi-Reihe zuzuordnen; da es nun im Prät. gebraucht wird, entspricht es den idg. Sekundärendungen, genauer der mi-Endung *-t-ām. Wenn wir weiter annehmen, wie ja der Sg., vor allem die 3. P. Sg., zu lehren scheint, dass der Aufbau der Endungen derselben Person mit Ausnahme des Anlautes sonst gleich war, dann muss in toch. Diphthong -ai- einfach eine hi-Endung der 3. Du. Akt. "-ăm stecken. ${ }^{24} \mathrm{Im}$ Aktiv scheint es also im Idg. ursprünglich folgende Endungen der 3. P. Du. gegeben zu haben:

$$
\begin{array}{lll} 
& m i-R e i h e & h i-R e i h e \\
\text { sekundär } & *_{-t-a} m & *_{-}-\bar{a} m \\
\text { primär } & *_{-t-e s} & *_{-e s ? ?}
\end{array}
$$

Es scheint nun, dass das hier angewandte Prinzip in der Opposition der $m i$-Reihe gegen die $h i$-Reihe in den Endungen der 3. Person auch auf die mediopassiven Endungen der 3. Du. appliziert

24 In einem anderen Aufsatz werde ich nachweisen, dass die idg. Auslautsgruppe ${ }^{*}-\bar{a} m$ zum historischen $-a i$ wird. Dadurch werden mehrere Geheimnisse der tocharischen sowohl nominalen wie verbalen Flexion aufgehoben. 
werden kann. Wir fanden oben, dass es einst Endungen dieser Person gab, die nur in einem Vokal, $*_{-\breve{l}, *} \breve{e}, *-\bar{e}$ bestanden. Nun war in der 3. Sg. in der mi-Reihe als sekundäre Medialendung *-t-ŏ gebraucht. Wenn wir nun die eben genannten Dualendungen ${ }^{*}-\check{\iota}$ usw. als zu derhi-Konjugation gehörig betrachten, ${ }^{25}$ was ja nahe liegt, da das -t- fehlt, so können wir als deren Schwesterformen in der mi-Konjugation gerade *-to- + diese Elemente erwarten, d. h. "to- $\ddot{i}>{ }^{*}-t o i$, was im indoiran. ${ }^{*}-t a i$ fortlebt. Aus *-to- + * $\breve{e} / \bar{e}$ wäre etwa ein *-tō zu erwarten, was sehr wahrscheinlich in ar. " -tăm steckt. ${ }^{26}$ Nun sind diese kurzen mi-Endungen nur selten gebraucht worden, wie wir oben sahen, hauptsächlich infolgendessen, weil sie entweder schon im Idg. (so *-toi) oder erst im Ar. (so *tôm>*-tām) mit anderen Endungen zusammenfielen. Andrerseits wurden sie je nach dem Auslaut in das bestehende System eingeordnet: *-toi wurde primär, da ja -oi sonst in primären Endungen steht, *-tôm blieb sekundär (und demzufolge noch mehr von aktivem *-tām bedroht!).

In der nächst folgenden Zeit entstanden aus Deutlichkeitsgründen

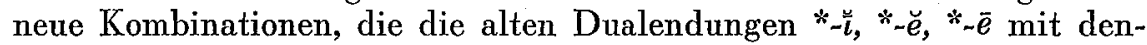
jenigen der $m i$-Reihe zu sog. langen Endungen vereinigten. ${ }^{27}$ Jede Möglichkeit des Zusammenfalls mit nichtdualischen Endungen wurde dadurch entfernt. Doch diese langen Endungen konnten nicht vollständig siegen: in sporadischen Fällen blieben neben ihnen die kürzeren der mi-Reihe bestehen. Das Indoiranische entfernte jedoch vollständig die ursprüngliche, aus einfachen nominalen Dualendungen bestehende Reihe.

Es stellt sich nun die Frage, wie die Endungen der 2. Du. Med. zustandekamen. Erinnert man sich an die oben schon besprochene Ten-

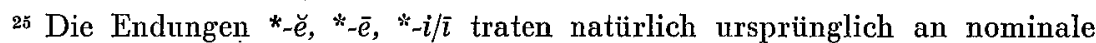
Stämme im Rahmen des Verbum finitum; rgl. oben. Wie das Perf. *rooid-e »er weiss zeigt, war solcher Nominalstamm auch in der hi-Konjugation bzw. im Perf., das mit der hi-Konjugation in der Wahl der Endungen zusammenging, in der 3. Sg. und wohl auch sonst anwesend. Das hi-Verbum *dheugh- »berihren (sich gut treffen), driicken, ausdrücken, melken, reichlich spenden « (Pokorny

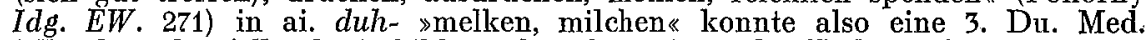

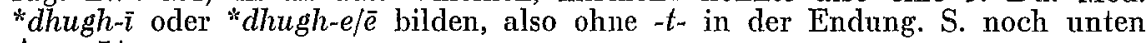
Anm. 34 .

${ }_{26}$ Das auslautende ${ }^{*}-m$ ist scheinbar ein fakultatives Element, das auch in einigen anderen Dualendungen vorzukommen scheint; so beim Verbum in der 3. Du. Akt. *-tām neben *-tă (das letztere im Bsl., sekundär auf die 2. Du. übertragen). Auch die 2. Du. Akt. ${ }^{*}$-to-m kann in *-to- + solches Element *-m zerlegt werden. - In der Nominaldeklination kommt solches * $m$ im Dat.-Abl.Instr. Du. vor: ar. -bhyäm neben *bhyä; vgl. auch air. di-bn sduobus« aus ${ }^{*}-b h x m$. Es ist jedoch völlig dunkel, welche Funktion dies *-m einst ausiubte; einfach ephelkystisch kann es von Haus aus nicht sein.

27 Ahnliche Kombinationen auch sonst in den Dualendungen; vgl. im Perf. Alkt.: ai -á-th-ur, -á-t-ur, av, -a-t-a-r, -ā-t-ar. Brugmann, Grdr.2 II 3 II, 657 sagt dazu: Mit ihrem dem Dentalformans vorausgehenden Vokal erinnern diese Dualformen (2. 3. Med.) an die aktivischen Dualendungen im Ind. Perf. ai. -áthuh, -átuh, av. -atar, und die Art der Entstehung mag beiderseits dieselbe gewesen sein. $\ll$ 
denz der 2. P. Du., an Stelle des -t- der 3. P. Du. das aspirierte $-t h$ - anzuwenden, so wäre die Antwort darauf sehr einfach. Doch hat es damit eine andere Bewandtnis. Zuerst muss man feststellen, dass die Vokale

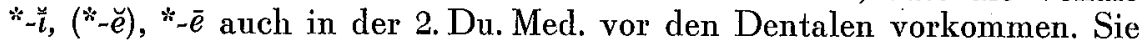
können nicht einfach aus der 3. Du. Med. herïbergenommen worden sein. Es scheint folglich, dass auch die 2. Du. Med, einst ganz dieselben Formen mit nominalen ${ }^{28}$ Dualendungen bildete wie die 3. Du. Med.: auf $*_{-\breve{u}},\left({ }^{*}-\breve{e}\right)$, und ${ }^{*}-\bar{e}$. Diesmal kann man kaum an gänzlich nominalen Ursprung der gesamten Formen denken, denn die 2. Du. ist ja eine verbale Form mit verbalen Mitteln, also mit einer verbalen Personenbezeichnung. Nun kann man aber schon wieder feststellen, dass es auch in der 2. Du. mehrere Reihen von Personalendungen gab:

a) die Reihe mit *-th- wurde schon oben besprochen und mit den Endungen der uibrigen 2. P. zusammengestellt; ${ }^{29}$.

b) sicherlich zur $h i$-Reihe gehörte eine Reihe von Personalendungen, die in den 2. P. im Dienste standen; ihr Zentralzeichen war ein Vokal, der Dental fehlte vollends: 1. 2. P. Pl. Perf. Akt. ai. -á (vid-á »ihr wisst usw.); 2. 2. P. Sg. Akt. primär *-ei (oder ${ }^{*}-\bar{e} i$ ?) ${ }^{30}$ in thematischen Präs.: gr. ̨̣épsı-ç, lit. vedì »du führst«; da der Zusammenhang mit der Endung unter 1. anzunehmen ist, muss man wohl von einer Vereinigung dreier Elemente reden: Themavokal *-e- + Personenzeichen *-e- + deiktisches (auf die Gegenwart hinweisendes) Element *-i, demnach am wahrscheinlichsten ein Langdiphthong *-ei mit Stosston (vgl. das Lit.); 3. im Hethitischen steht im Mediopassivum in der 2. P. Sg. im Prät. neben -tat auch -at: kiš-tat neben kiš-at von kiš- »werden «; da kiš- der hi-Konjugation angehört (vgl. 3. Sg. Präs. kiš-ari, kiš-a!), wird man auch hier an etwas Altes denken müssen; ${ }^{31}$ es ist kaum glaublich, dass es sich hier um eine

${ }^{28}$ Dieser Ausdruck ist nur vom Standpunkt des Indogermanischen kurz vor der Auflösung der Gemeinschaft richtig. Einst waren aber die verbalen wie die nominalen Numeruszeichen gleich, s. die Anm. 23.

${ }^{20}$ Die $t h$-Endungen der 2. Du. sind z. T. der hi-Konjugation oder besser dem Perfekt zuzuschreiben, vgl. "-tha in der 2. Sg. Akt. des Perfekts. Es ist aber auch möglich, dass die th-Endungen in den Dualformen sekundär in der hiKonjugation aufkamen, um das System zu vervollkommnen, und zwar erst durch den Ersatz eines -t- der mi-Endungen durch das -th- der hi- und Perfektendungen.

${ }_{80}$ Gr. phérei-s (mit $-s$ erweitert) $=$ air. -bir »fers«. Lit. z. B. bei Schwyzer, Gr. Gr. I 661. Über die lit. Endung heute anders Stang, Vergl. Gramm. 407, der von urbalt, -ai ausgeht, das er im Altlit. findet; danach Watkins, Idg. Gr. III 1, 212, der S. $213 \mathrm{f}$. dafür ein idg. ${ }^{*}-O-i$ aufstellt. Das altlit. -ai kann jedoch nur eine Nebenform zu*-ēi sein. Der blosse Vokal als Zeichen für die 2. P. scheint jedoch $\mathrm{m}$. E. zu bleiben.

${ }_{31}$ Neu, Das heth. Mediopassio und seine idg. Grundlagen 28 nennt nur ešrat, kišs-at. Daraus könnte man vielleicht erschliessen, dass dies idg. mediale ${ }^{*}-o$ nur dem Perfekt angehörte, erst hiervon in das allgemeine Präteritum des Anatolischen eindrang.

Beim Präs. nennt Neu die 2. Sg. Med. karuššj-ari (von karuššija- »schweigen; gleichgültig sein, ruhig zusehen «). Er fährt S. 17 fort: »hierbei handelt es sich m. E. nicht um einen Schreibfehler, sondern die 3. sg. steht hier an Stelle 


\section{Bojan Čop}

Umbildung nach der 3. Sg. Prät. (-at) handle; wie die idg. Urform aussah, ist jedoch ziemlich schwer zu erraten (etwa *o, denn -t(i) ist erst heth. Zutat). Trotz aller möglichen Einwände ist es jetzt klar, dass eine Reihe von Personalendungen, die nur aus einem Vokal bestand (woran Numerus- und Diathesenzeichen, die z.T. schon ganz verdunkelt sind, traten), auch in der 2. Sg. und Pl. der hi-Konjugation vorkam; eine Annahme derselben Reihe auch für den Dual ist nur ein kurzer, aber berechtigter Schritt weiter vorwärts. Somit können für den Dual Med.

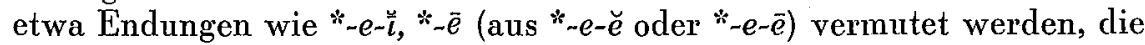
für die 2.P. gebraucht wurden.

Auch diese Endungen waren mehrdentig, so dass man auch hier nach den eindeutigen Kombinationen mit den th-Endungen trachtete. ${ }^{32}$ So kamen endlich die längeren Endungen des historischen Indoiranisch zustande.

Hier muss man die toch. Endung der 2. P. Du. Imper. Med. -ai-t einreihen. Wir haben schon oben festgelegt, dass das -a- vor dem zweiten Bestandteil des Diphthonges wohl nicht idg. Themavokal -e- (oder sogar -o-) ist, sondern dass es sich in diesem - a- um den dem Imperativ eigenen Themavokal tocharischen Ursprunges handelt. Es ist also zu fragen, wie eigentlich die toch. Endung *-a-i zustande kam. Es muss einst auch ein indikativisches ${ }^{*}-e-\bar{\imath}$ (gemäss den obigen Feststellungen), das den athematischen Systemen angehörte, gegeben haben; hier wurde -e- später als Themavokal aufgefasst, so dass im Imper. der Ersatz desselben durch ein jüngeres - a- möglich war. Leider hat uns das Tocharische noch keine indikativische Endung für die 2. Du. Med. geliefert, die allein imstande wäre, obige Vermutung zu bestätigen.

Auch im Arischen muss das urspr. der athematischen Konjugation angehörende *-e-i später: als thematisch aufgefasst worden sein.

Es ist sehr wahrscheinlich, dass einst die drei kürzeren Endungen,

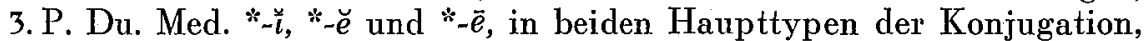
im thematischen und athematischen, gleichmässig vorkamen; denn wir fanden oben, dass im Altindischen $-\bar{a}-t h \bar{e}$ im Konj. vorkommt und der Konjunktiv ist äusserlich ein thematischer Konjugationstypus; ebenso kam - $\bar{a}-t h a \bar{a} m \mathrm{im}$ thematischen Inj. = später Imper. vor. Anderseits

der 2. Sg. Ähnlich zu beurteilen sind die Präteritalformen ešat und kišat (3. Sg.), die auch in der 2. sg. Verwendung finden. « Dem entgegenhalten kann man, dass heute, als blosser Vokal als Zeichen der 2. Sg. auch sonst vorkommt, die Erklärung als 3. Sg. kaum in Betracht kommt. Auch das Hethitische hat demnach schwache Spuren des alten Zustandes in der 2. Sg. erhalten. -ari also =idg. dial. $* 0-r-i$.

${ }^{32} \mathrm{Zu}$ betonen ist, dass es oben im Text festgestellt wurde, dass in der 2. P. Du. keine längere Endung mit dem kurzen -a- vor dem Dental im Arischen zu finden ist. Obwohl diese Person im Avestischen, wo allein sie auch mit kurzem -a* erscheinen könnte, sehr schwach belegt ist, kann dieser Umstand doch wichtig sein: die kürzeren Endungen, bestehend aus einem Vokal ohne Dental, waren hier nur ${ }^{*}-e-i$ und ${ }^{*}-\bar{e}$, eben deshalb, weil hier noch das Personenzeichen

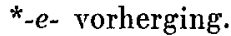


kommt -i-tam im Avestischen in athematischer sowie thematischer Flexion vor. Die urspr, Dualzeichen waren in dieser Hinsicht indifferent. Die andere Frage, ob sie hinsichtlich der $m i$ - und $h i$-Konjugation ebenso indifferent waren, ist schwieriger; in der 3. Du. scheint keine Differenz dieser Art fortzuleben, ebensowohl in der 2. Du., wenn die th-Endungen, insoweit überhaupt für die Ursprache vorstellbar (das -th- kann ja erst eine arische Neuerung sein), der mi-Konjugation angehörten, was eben sehr leicht bestreitbar ist.

Wenn wir jetzt versuchsweise, ohne auf volle Güiltigkeit der Voraussetzungen Anspruch zu erheben, eine Tabelle aufstellen, so haben wir:

\begin{tabular}{|c|c|c|}
\hline \multirow{4}{*}{$\begin{array}{l}\text { 3. P. Du. Med. } \\
\text { 2. P. Du. Med. }\end{array}$} & \multicolumn{2}{|c|}{ hi-Konjugation } \\
\hline & primär & sekundär \\
\hline & $-\breve{l},-\breve{e},-\bar{e}$ & $-\breve{l},-\breve{e},-\bar{e}$ \\
\hline & $\begin{array}{l}-e-\breve{i}>-e i,-\vec{e} \\
-t h a^{x}-\breve{l}^{33}\end{array}$ & $\begin{array}{l}-e-\check{l}>-e i,-\bar{e} \\
-t h \bar{o}-m\end{array}$ \\
\hline & \multicolumn{2}{|c|}{ mi-Konjugation } \\
\hline $\begin{array}{l}\text { 3. P. Du. Med. } \\
\text { 2. P. Du. Med. }\end{array}$ & $\begin{array}{l}- \text { to }-\breve{\imath}>- \text { to } \\
\text {-tho }-\bar{l}>- \text { thoi? }\end{array}$ & $\begin{array}{l}-t \bar{o}-m \\
-t h \bar{o} \sim m ?\end{array}$ \\
\hline
\end{tabular}

Es ist verständlich, dass manche Frage hier unbeantwortet blieb; es gibt ja Probleme, die niemals Lösung finden werden; dazu ist eben das uns zur Verfügung stehende Material zu gering. So ist es hier mit der th-Endung bewandt: man weiss nicht, ob sie in beiden Hauptkonjugationen gebildet wurde oder nicht, wie weit sie in der hi-Konjugation verbreitet war u. a. ${ }^{34} 35$

${ }_{33}$ Die Vokalfarbe ist hier unsicher; man hat in den th-Endungen -a in *-tha, -e- in *-thēs festgestellt. Doch konnte daneben auch ein mediopassives*-thostehen (vgl. die 3. P.).

${ }^{34}$ Man könnte weiter vermuten, dass es einst in einigen Formen des Duals, so in der 3. P., die nominalen Ursprunges ist (s. oben im Text), ähnlich wie im Semitischen, einen Genusunterschied gab; im Indogermanischen hätte man hier die Opposition animé (Mask. + Fem.): inanimé (Ntr.), die mit der Opposition in den zwei Endungen $-e:-\bar{\imath}$ verwirklicht wurde: *dhugh-é (animé): *dhugh-ī' (inanimé). So würde sich am besten die etwas stutzig machende Buntheit der Dualendungen erklären lassen.

Die Opposition animé: inanimé wurde schon früher in den Personalendungen der 3. Pl. festgestellt: Pedersen hat z. B. so die Opposition $-n t-$ : $-r$ zu erklären versucht, z. B. Hittitisch 86 (er wendet nur andere Ausdrücke an).

${ }^{35}$ Die bisherigen Erklärungen dieser Endungen sind kaum anzuerkennen, da sie die toch. Endung -ai-t nicht in Betracht ziehen.

Hirt, Idg. Gr. IV 157 behandelt ganz kurz die Endungen mit $-\bar{a}-$ des Arischen; sie sollen auf Umbildungen beruhen. $\$ 50$, S. 106 bespricht er die 1 . Sg. Med. ai. auf $-\bar{e}$ (Präs., Perf.), auch als 3. Sg. gebräuchlich, und betrachtet sie als urspr. Infinitiv; er fährt fort: $»$ Wahrscheinlich liegen sie (d. h. Infinitive) auch der 2. und 3. Du. zugrunde, da man ai. 2. Du. Med. Präs. bhávēthē, 3. Du. 
Der vorliegende Aufsatz wurde im J. 1963 verfasst. Dies ist nötig, um zu verstehen, warum auf Ausführungen von Kuryłowicz, Inflect. Categories (1964), S. 153-155, kein Bezug genommen wurde: einfach darum, weil diese strukturalistischen Manipulationen der Tatsachen für einen Logiker ohne Überzeugungskraft sind. Ich füge hinzu, dass das -ai- der toch. Dualendungen im Akt. und Med. mit dem -é- der ai. Dualendungen vermutungsweise schon vor mir bei Watkins, Idg. Gr. III, 1. Teil (1969), S. 48 verbunden wurde, jedoch ohne jede Erörterung des Ursprunges und der einstigen Funktion dieser Diphthonge. Wie im Text gesagt, ist aktives -ai- ganz unterschiedlichen Ursprunges.

\section{Poozetek}

\section{K 2. IN 3. OS. DVOJINE MEDIOPASIVA V IEVR.}

Sti., av., gr. in tohar. imajo za v naslovu omenjeni osebi svojske končnice. Grške so zanesljivo novotvorbe brez vrednosti za prajezik. Preostanejo torej sti., av. in tohar. oblike.

$\checkmark$ klas. sti. so bile končnice za ti dve osebi popolnoma enovite in sistemsko

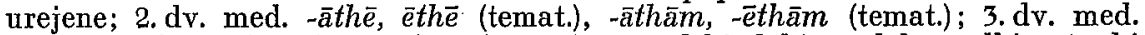
$-\bar{a} t \bar{e},-\bar{e} t \bar{e}$ (temat.), -ātām, -êtām (temat.); v vedski dobi se dobe redki ostanki drugačnih končnic: 3. dv. med -ittām tudi atem., 2. dv. med, thām, the. To

bhávē-tē, 2. Du. Med. Imperf. á-bhavē-thām, 3. Du. ábhavêtām aus bhavēe + angetretene Partikel wird erklären müssen.«

Hirt in Thumb, $\mathrm{Hb}$ d. Sanskrit ${ }^{2}$ I 507, Nachtr. zu $\$ 432: » M$. E. liegt den Formen wie doišăthé, bharēthe eine Art Stammform (Infinitv doiša und bharēe) zugrunde, an die deiktische Elemente angetreten sind.ณ

Das Operieren mit funktionslosen Stammformen und Infinitiven ist natïrlich ein Verfahren ohne Nutzen.

Burrow, The Sanskrit Language (2nd impr. 1959) $312 \mathrm{f}$. sagt: »The IndoIranian forms are connected with the corresponding active terminations of the dual. The same variation between th and $t$ appears between the two persons in the primary endings, and in the middle this is introduced also into the secondary endings. The final $-e$ of the primary endings is the same $-e$ characterising the middle which appears throughout the primary system. The influence of this $-e$ of the middle is responsible for the substitution of $e$ for the a of thematic stems (ábhavetām as opposed to active ábhavatām, Av. jasaētom beside jasatom). Non thematic verbs are distinguished by an $\bar{a}$ of the middle ending, but examples like Av. dazde 3. du. pf. and Skt. cikethe indicate that this is an innovation. It can only have come from formations of roots in $\bar{a}$ like dad $\vec{a}{ }^{\prime}$ the, dad $\bar{a}^{\prime} t e$ where the $\bar{a}$ is originally part of the root as in 2. sg. dada'tha (beside dadithá) and 2. pl. dádhātana (beside dhattana).«

Burrow lässt in dieser Erklärung die Formen mit $-\bar{\imath}$ - und kurzem -a- ausser Acht. Das $-\bar{e}-$ der thematischen Konjugation kann ja nicht so einfach in das Innere der Formen vordringen, zumal es auch in den selcundären Formen steht. Auch das - $\bar{a}$ - kann kaum wurzelhaft sein, denn gerade seine Dubletten mit und ohne - $\bar{a}-$ zeigen, dass es nach der Ansicht der Alten sicher zur Wurzel gerechnet wurde; auch die Statistik spräche dagegen.

Vgl. noch Wackernagel, Altind. Gr. I, Nachtr. von Debrunner S. 52, wo D. adhitâm mit $a-d h \bar{i}-m a h i$ vergleicht und entsprechend av. da $a^{i} \delta \bar{i}-t a m$ analysiert. Er zitiert noch Pisani, Gr. $\$ 508$, der e/ā als nach dem Muster der Optative bhavetām doišy $\bar{a} t \bar{a} m$ entstanden sein lässt. Kaum anzunehmen. 


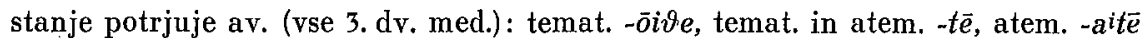

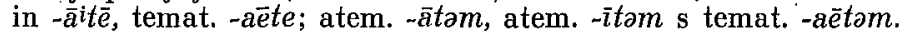

Vse to skupaj daje misliti, da so ustrezne končnice bile nekoč razvrščene po tem, kaj stoji pred dentalom, v štiri skupine: a) končnice brez vokala pred dentalom, b) končnice s kratkim -a- pred njim (zabeležena le končnica *a-tai), c) končnice $\mathrm{z}$ dolgim - $\bar{a}$ - pred dentalom, d) končnica $\mathrm{z}-\bar{i}-$ pred dentalom. $\mathrm{V}$ av. se je v selkund. končnicah stari -ām nadomestil $\mathrm{z}$-ăm, pač po akt. -tom iz -tam.

Večina elementov, ki naše končnice sestavljajo, se da z lahkoto razložiti: a) -th- v 2. dv. ima ob sebi isti konsonant v 2. os.: *-thes za 2. dv., *-tha v 2. edn. perf. akt., "thess za 2. edn. med. sekund.; vsaj v ar. še *-the za 2. Pl. Akt. primar.; -t- za 3.os. je splošno znan, v direktni opoziciji s -th-za 2. os. stoji $\mathrm{v}$ ar, ravno $\mathrm{v}$ du. akt. (-thas: -tas, $\mathrm{v}$ perf. -áthur:-átur); b) končni diftong $\mathrm{v}$ *thai, *tai se sklada $z$ diftongom v medialnih končnicah (primar) 1. edn. "-axi, 2. edn. *-soi, 3. edn. *-toi, 3. mn. *-ntoi itd.

Torej moramo razložiti le vokalne elemente pred dentali: ar. $-a-,-\bar{a}-$ in $-\bar{\imath}-$. Tu moramo priklicati na pomoč toharščino. V njenem dialektu B se je ohranil edinstveni primerek končnice za 2 . dv. imper. med. -ait v p-yam-tts-ait »naredita!«; tu je -t identičen s končnico 2. mn. med.: p-yām-tsa-t »naredite! « in izvira iz ievr. *-dhu(m). Diftong -ai- pred tem je sestavljen iz imper. znaka -ain $-i \sim$, ki ga smemo identificirati $z-\bar{l}-\mathrm{v}$ ar. dvojinskih končnicah zgoraj.

Ce preostala vokala dvojinskih končnic rekonstruiramo $\mathrm{v}$ ievr. ${ }^{*} e-$ in ${ }^{*}-\bar{e}$-, dobimo trojico vokalnih elementov, ki so identični $z$ ievr. nominalnimi dvojinskimi znaki: * $\breve{e}^{*}{ }^{*}-\bar{e}$ in ${ }^{*}-\bar{l}$. Del oblik verbi finiti je bil itak nominalnega izvora, tako zl. v 3. os. Omenjene dvojinske nominalne končnice so pripadale prv. tkim. hi-jevski konjugaciji, kjer so se pridevale na glagolsko deblo brez dentalov. Vzporedne $\mathrm{s}$ temi so bile $m i$-jevske dvojinske končnice, ki so vsebovale dentale, v 3 . os. $-t-$, tako da so imeli končnici ${ }_{-} t o-\bar{\imath}$ in ${ }^{*}-t \bar{o}(m)$. Ker so te kratke končnice sovpadle $\mathrm{z}$ drugofunkcionalnimi, so $\mathrm{z}$ novo metodo to preprečili: $\mathrm{s}$ $h i$-jevskimi starimi končnicami so združili $m i$-jevske $\mathrm{v}$ tkim. dolge končnice, torej $*_{-i}-$ toi itd.

Podoben je bil razvoj v 2. dv. med.: Tu so poznali nekoč dve skupini končnic: eno s centralnim znakom -th-, drugo s centralnim znakom kratek vokal brez dentala (prim. 2. mn. perf. akt. sti. -á, 2. edn. akt. primar. "-ēi; oboje je spadalo $\mathrm{v}$ hi-jevsko konjugacijo), tako da smo imeli tu *-tho-i, *tho(m) in ${ }^{*}-e-i,{ }^{*}-\bar{e}$. Tu smemo vključiti še toh. končnico -ai-t: ta je nastala ob posnemanju indikativnega ${ }_{-}-e-i$, $\mathrm{kar}$ je spet s svoje strani postalo kasneje $\mathrm{v}$ ar. in toh. del sistema tematskih glagolov. Kmalu so s kombinacijo obeh tipov nastale še tkim. dolge končnice, ki so dokoněno preprečile zamenjavo $z$ drugimi osebạmi. 